\title{
Relationship Between Physical Performance and Mild Cognitive Impairment in Chinese Community-Dwelling Older Adults
}

This article was published in the following Dove Press journal: Clinical Interventions in Aging

\author{
Yuewen $\operatorname{Liu}^{1}, *$ \\ Weibo $\mathrm{Ma}^{2}$,* \\ Ming $\mathrm{Li}^{3}$ \\ Peipei Han' \\ Ming Cai' \\ Feng Wang ${ }^{2}$ \\ Jingru Wang ${ }^{2}$ \\ Xiaoyu Chen' \\ Jianrong $\mathrm{Shi}^{4}$ \\ Xiaoyan Zhang ${ }^{4}$ \\ Yiyi Zheng' \\ Mengqiu Chen' \\ Qi Guo (D)' \\ Ying Yu'
}

'Department of Rehabilitation Medicine, Shanghai University of Medicine and Health Sciences, Shanghai, People's Republic of China; ${ }^{2}$ Department of Nursing and Health Management, Shanghai University of Traditional Chinese Medicine, Shanghai, People's Republic of China; ${ }^{3}$ Office of the President, Shanghai University of Medicine and Health Sciences Affiliated Zhoupu Hospital, Shanghai, People's Republic of China; ${ }^{4}$ Department of Team, Buzhen Community Health Service Center, Chongming District, Shanghai, People's Republic of China

*These authors contributed equally to this work

\begin{abstract}
Objective: This study aimed to examine the relationship between physical performance and mild cognitive impairment (MCI) in Chinese older adults.

Methods: The sample comprised 956 relatively healthy and aged $\geq 65$ years old Chinese community-dwelling participants (mean age, $72.56 \pm 5.43$ years; $56.8 \%$ female), which did not include those with dementia, severe cognitive impairment, mental illness etc. The MiniMental State Examination (MMSE) and the Instrumental Activities of Daily Living (IADL) scale were used for the initial classification of patients with MCI. Physical performance was measured via hand grip, Timed Up and Go Test (TUGT), and 4-m walking speed.

Results: The physical performance (grip strength, TUGT, and 4-m walking speed) correlated with MCI. The grip strength [odds ratio $(\mathrm{OR})=0.96,95 \%$ confidence interval $(\mathrm{CI})=$ $0.93-0.99$ ] and 4-m walking speed $(\mathrm{OR}=0.25,95 \% \mathrm{CI}=0.10-0.64)$ correlated negatively with $\mathrm{MCI}$, while TUGT ( $\mathrm{OR}=1.08,95 \% \mathrm{CI}=1.03-1.13)$ and MCI correlated positively.

Conclusion: The physical performance (grip strength, TUGT, and 4-m walking speed) correlated with MCI. Further analysis showed that the grip strength was associated with overall cognition, time orientation, recall, and language, while TUGT and 4-m walking speed were associated with overall cognition and various cognitive domains, except recall.
\end{abstract}

Keywords: mild cognitive impairment, grip strength, TUGT, 4-meter walking speed

\section{Introduction}

Age-related cognitive decline has become a public health concern with the rise in the aging population all over the world. Mild cognitive impairment (MCI) has been conceptualized as an intermediate stage between normal aging and dementia. ${ }^{1} \mathrm{MCI}$ is considered as a risk factor for dementia. ${ }^{2}$ Older adults with MCI develop dementia at a rate of $10 \%-15 \%$ every year, ${ }^{3}$ and at a rate of $60 \%-100 \%$ within $5-10$ years. ${ }^{4}$ However, one third of patients with MCI remain in clinical stability or even return to normal. ${ }^{5}$ Therefore, the study of MCI is of great significance for the prevention of dementia. At present, no pharmacologic treatments are available to cure the progression of MCI to dementia. Hence, the prevention of the deterioration of MCI to dementia is very important. Early detection of those most at risk is necessary to appropriately target early treatments, but the current screening approaches are too invasive and costly to be used at a population level. Accordingly, early clinical indicators of risk are needed.

The physical function of the older adults gradually decreases with the increase in age, and their physical performance is restricted to varying degrees. Cognitive
Correspondence: Qi Guo; Ying Yu Email guoqijp@gmail.com;

yuying@sumhs.edu.cn 
function is related to prefrontal cortex, parietal lobe, and hippocampus, which affect the physical performance of individuals. ${ }^{6-8}$ A study underscored the importance of executive function (dorsolateral frontal cortex) and memory function (hippocampus) in gait control in older adults. ${ }^{6}$ Gait decline increases the risk of cognitive decline and dementia. ${ }^{7}$ Poor mobility outcomes were reliably associated with reduced gray and white matter volume. ${ }^{8}$ At present, the evaluation of the physical performance of older adults mainly focuses on mobility, balance, and muscle strength. Several studies showed that mobility, balance, and grip strength were associated with changes in cognitive function. ${ }^{9-11}$ However, views on the relationship between balance, muscle strength, and cognition are still inconsistent. Some studies found that poor balance performance correlated with cognitive impairment, ${ }^{10}$ but others found that balance did not correlate with cognition. ${ }^{12}$ Some studies found a relationship between handgrip strength and global cognitive performance, while the relationship between other cognitive domains needs further research. ${ }^{13}$ Very few studies examined the relationship between mobility, balance, grip strength, and cognition in older adults; however, the conclusions were inconsistent.

Therefore, this study aimed to examine the relationship between physical performance and $\mathrm{MCI}$ in Chinese older adults. Moreover, it also investigated whether physical performance (strength, balance, and mobility) was related to specific cognitive functions. Therapeutic interventions specifically aimed at maintaining or improving one or more physical performances might help slow down a decline in condition or even to improve cognitive functioning in cognitively impaired older people. The data of the study might provide basic scientific and theoretical support in the strategy and policy making for the prevention of MCI and Alzheimer's disease (AD) in China.

\section{Methods}

\section{Study Population}

The study population is from Chongming District, Shanghai. All subjects were invited to participate in a comprehensive geriatric assessment and cognitive function assessment. Inclusion criteria: (1) Older adults people $\geq 65$ years old; (2) Have lived in the community for at least one year; (3) Willing to participate in this study. Exclusion criteria: (1) severe cognitive impairment, dementia, mental illness or other neurodegenerative diseases; (2) those who could not take care of themselves and the instrumental activities of daily living scale were more than or equal to three items that could not be completed and could not walk independently; (3) people with hearing impairment, visual impairment and communication difficulties; (4) those who took sedatives in the last one or two months; (5) unable to perform the grip strength, Timed Up and Go Test (TUGT), and 4-meter walking test. The final study population comprised 956 subjects. All participants provided informed consent prior to participation.

\section{Assessment of Cognitive Function}

The Mini-Mental State Examination (MMSE) was used for the initial classification of patients with MCI to determine their needs in a further and comprehensive assessment. ${ }^{14}$ Therefore, we used the brief cognitive test to assess cognitive function. Cognitive assessment was completed using the MMSE $^{15}$ by trained investigators. It includes 30 items, the score ranges from 0 to 30 points, with the higher scores indicating better cognitive performance. The MMSE includes a broad set of cognitive domains that measure the following: orientation to time (5 points), orientation to place ( 5 points), registration ( 3 points), attention and calculation ( 5 points), recall ( 3 points), and language ( 9 points). ${ }^{16}$

\section{Assessment of Daily Activity Ability}

The daily activity ability was tested using the Instrumental Activities of Daily Living scale (IADL). ${ }^{17}$ It includes 8 items, the score ranges from 0 to 8 points, with the higher scores indicating better daily activity ability.

\section{Definition of $\mathrm{MCl}$}

The definition was made according to the diagnostic criteria of Petersen. ${ }^{18}$ The MMSE and IADL to definition MCI, that is MMSE to determine cognitive impairment and IADL judged normal daily activities ability. The cut-off points used for cognitive impairment were as follow: $\leq 17$ for illiterate people, $\leq 20$ for people with primary school, and $\leq 24$ for people with middle school or higher. ${ }^{19}$ IADL scores $\geq 6$ indicates normal daily activity ability. ${ }^{20}$

\section{Physical Performance Assessment: Hand Grip, TUGT, Four-Meter Walking Test}

Hand grip was measured using a dynamometer (GRIP-D; Takei Ltd, Niigata, Japan). Participants were asked to exert their maximum effort twice using their dominant hand, the mean grip strength was calculated from two attempts. ${ }^{9}$ The TUGT beings with the participant sitting on a chair in 
a relaxed position, getting up from the chair, then walking 3 meters, turning around, walking back to the chair, and then sitting down chair. ${ }^{21}$ Walking aids were allowed. The 4-meter walking test consists of participants being timed while walking four-meter at their usual pace. Participants completed the test twice and the mean gait speed $(\mathrm{m} / \mathrm{s})$ was calculated. ${ }^{9}$

\section{Covariates}

All participants were invited to a face-to-face interview to answer a standardized questionnaire. The questionnaire included questions about age, sex, height, body weight, occupation, illiterate, living alone, sleep duration, smoking habits (current smoker, never smoking, and past smoker), and drinking habits (drinking daily, occasional drinking, past drinking, and never drinking). Physical activity was assessed using the short form of the International Physical Activity Questionnaire (IPAQ). ${ }^{22}$ A history of physical illness was evaluated based on participants' responses (yes or no) to questions about their history, past diagnoses made by physicians, and current or historical medication regimens. Diseases of interest included type 2 diabetes mellitus (T2DM), hypertension, hyperlipidemia, gout, anemia, pulmonary disease, biliary tract disease, kidney disease, peptic ulcer, hepatic disease, heart disease, osteoarthritis, cancer and thyroid disease.

\section{Statistical Analysis}

Continuous variables were expressed as mean \pm standard deviation; continuous nonnormal distribution variables, such as IPAQ, were expressed as median and quartile; and classified variables were expressed as a percentage (\%). Differences in baseline characteristics according to cognitive status were analyzed using the $t$ tests, Pearson's chi-square test, and Mann-Whitney $U$-tests. Logistic regression models were used to examine the relationship between physical performance and MCI. Linear regression models were used to analyze the relationship between grip strength, TUGT, walking speed, overall cognition, and various fields. Model 1 adjusted variables included age, sex, and body mass index (BMI), and Model 2 adjusted variables included age, sex, BMI, IADL, illiterate, widow, living alone, diabetes, hypertension, hyperlipidemia, and hepatic disease. All the statistical analyses were performed using SPSS version 21.0, and a $P$ value less than 0.05 was considered statistically significant.

\section{Ethics}

The study was approved by the Ethics Committee of Shanghai University of Medicine and Health Sciences and the methods were carried out in accordance with the principles of the Declaration of Helsinki.

\section{Results \\ Characteristics of the Participants}

In total, 956 individuals participated in the study (mean age, $72.56 \pm 5.43$ years; $56.8 \%$ women), of which 47 individuals were excluded due to the submission of an incomplete questionnaire. Of the remaining participants, 138 (14.4\%) met the diagnostic criteria and were defined as having MCI. Table 1 presents the characteristics of these participants. Participants with MCI had lower daily activity ability and level compared with those with normal cognition. Compared with participants with normal cognition, those with MCI were older, more likely female, illiterate, widow, living alone, and suffering from hypertension; and had lower physical performance (lower grip strength, walking speed, and worse balance) and longer sleep time. Fewer patients suffered from the hepatic disease.

\section{Relationship Between Physical Performance and $\mathrm{MCl}$}

The relationship between physical performance and MCI is shown in Table 2 . In the fully adjusted model, the grip strength [odds ratio $(\mathrm{OR})=0.96,95 \%$ confidence interval $(\mathrm{CI})=0.93-0.99]$ and 4-m walking speed $(\mathrm{OR}=0.25$, 95\% CI $=0.10-0.64)$ correlated negatively with $\mathrm{MCI}$; larger grip strength and faster walking speed were the protective factors of $\mathrm{MCI}$. TUGT $(\mathrm{OR}=1.08,95 \% \mathrm{CI}=$ 1.03-1.13) correlated positively with MCI; slower TUGT was a risk factor for MCI.

Further, the relationship between grip strength, TUGT, 4-m walking speed, overall cognition, and various fields was analyzed. The grip strength correlated with the total score of MMSE, time orientation, immediate recall, and language (Table 3); and TUGT and 4-m walking speed correlated with the total score of MMSE, time orientation, place orientation, registration, attention and calculation, and language (Tables 4 and 5).

\section{Discussion}

In this suburb-dwelling older adults, the prevalence of MCI in people aged more than 65 years was $14.4 \%$ 
Table I Baseline Characteristics of Study Participants with Normal Cognition vs Mild Cognitive Impairment

\begin{tabular}{|c|c|c|c|}
\hline \multirow[t]{2}{*}{ Variables } & \multirow{2}{*}{$\begin{array}{l}\text { Normal Cognition } \\
(n=8 \mid 8)\end{array}$} & \multirow{2}{*}{$\begin{array}{l}\text { Mild Cognitive Impairment } \\
(n=\mid 38)\end{array}$} & \multirow[t]{2}{*}{$P$ value } \\
\hline & & & \\
\hline Age (year) & $72.00 \pm 5.03$ & $75.86 \pm 6.45$ & $<0.001$ \\
\hline Female (\%) & 54.6 & 69.6 & 0.001 \\
\hline BMI $\left(\mathrm{kg} / \mathrm{m}^{2}\right)$ & $23.62 \pm 3.49$ & $23.82 \pm 3.51$ & 0.535 \\
\hline IADL (score) & $7.79 \pm 0.49$ & $7.54 \pm 0.74$ & $<0.001$ \\
\hline MNA-SF (score) & $12.80 \pm 1.40$ & $|2.67 \pm| .4 \mid$ & 0.325 \\
\hline GDS (score) & $5.43 \pm 4.37$ & $7.11 \pm 5.57$ & $<0.001$ \\
\hline Sleep duration (hour) & $8.48 \pm 1.47$ & $9.01 \pm 1.64$ & $<0.001$ \\
\hline IPAQ (Met/wk) & $5150(2079,10,194)$ & $3764(1245,8400)$ & 0.012 \\
\hline Illiterate (\%) & 9.8 & 38.4 & $<0.001$ \\
\hline Widow(\%) & 18.7 & 42.0 & $<0.001$ \\
\hline Living alone (\%) & 15.5 & 30.4 & $<0.001$ \\
\hline Farming (\%) & 50.9 & 68.6 & 0.055 \\
\hline Smoking (\%) & & & 0.112 \\
\hline Current smokers & 16.2 & 13.0 & \\
\hline Never smokers & 66.6 & 75.4 & \\
\hline Ever smokers & 17.3 & 11.6 & \\
\hline Drinking (\%) & & & 0.379 \\
\hline Daily drinkers & 14.6 & 15.2 & \\
\hline Occasional drinkers & 14.5 & 14.5 & \\
\hline Former drinkers & 13.2 & 8.0 & \\
\hline Never drinkers & 57.7 & 62.3 & \\
\hline Grip strength (kg) & $23.54 \pm 8.91$ & $18.60 \pm 9.04$ & $<0.001$ \\
\hline 4-meter walking test $(\mathrm{m} / \mathrm{s})$ & $I .1 I \pm 0.23$ & $0.94 \pm 0.26$ & $<0.001$ \\
\hline TUGT (s) & $9.90 \pm 3.43$ & $12.70 \pm 5.20$ & $<0.001$ \\
\hline \multicolumn{4}{|l|}{ Diseases (\%) } \\
\hline Diabetes (\%) & 16.5 & 12.3 & 0.212 \\
\hline Hypertension (\%) & 61.9 & 75.4 & 0.002 \\
\hline Hyperlipidemia (\%) & 29.3 & 29.0 & 0.949 \\
\hline Gout (\%) & 6.4 & 6.5 & 0.944 \\
\hline Anemia (\%) & 4.5 & 7.2 & 0.172 \\
\hline Pulmonary disease (\%) & 8.3 & 8.0 & 0.890 \\
\hline Biliary tract disease (\%) & 14.6 & 18.1 & $0.28 I$ \\
\hline Kidney disease (\%) & 12.0 & 8.0 & 0.169 \\
\hline Peptic ulcer (\%) & 15.1 & 11.6 & 0.284 \\
\hline Hepatic disease (\%) & 18.1 & 8.7 & 0.006 \\
\hline Heart disease (\%) & 33.2 & 41.3 & 0.063 \\
\hline Thyroid disease (\%) & 3.8 & 6.5 & 0.140 \\
\hline Osteoarthritis (\%) & 16.2 & 21.0 & 0.159 \\
\hline Cancer (\%) & 3.8 & 2.2 & 0.342 \\
\hline
\end{tabular}

Abbreviations: BMI, body mass index; MNA-SF, mini-nutritional assessment short-form; GDS, geriatric depression scale; IADL, instrumental activity of daily living; IPAQ, international physical activity questionnaires; TUGT, timed up and go test.

with Petersen's diagnostic criteria. This finding was similar to a study showing that the prevalence of MCI was $14.71 \%$ in an older population. ${ }^{23}$ The present study examined the association between physical performance and MCI of the older adults in the suburb dwelling of China. The results of this study provided evidence that after adjustments for potential confounders, physical performance still correlated with MCI. Moreover, greater grip strength and faster walking speed were the protective factors for MCI, while slower TUGT was the risk factor for MCI. These results were consistent with previous findings. 
Table 2 Logstics Regression Analysis of Physical Performance and $\mathrm{MCl}$

\begin{tabular}{|c|c|c|c|c|c|c|}
\hline \multirow[t]{2}{*}{ Variables } & \multicolumn{2}{|l|}{ Unadjusted } & \multicolumn{2}{|l|}{ Model I } & \multicolumn{2}{|l|}{ Model 2} \\
\hline & OR (95\% Cl) & $P$ & OR (95\% Cl) & $P$ & OR (95\% Cl) & $P$ \\
\hline Grip strength & $0.93(0.91-0.95)$ & $<0.001$ & $0.95(0.92-0.98)$ & 0.001 & $0.96(0.93-0.99)$ & 0.015 \\
\hline TUGT & 1.15 (1.10-1.2I) & $<0.001$ & $1.11(1.06-1.16)$ & $<0.001$ & $1.08(1.03-1.13)$ & 0.002 \\
\hline 4-meter walking test & $0.06(0.03-0.13)$ & $<0.001$ & $0.15(0.07-0.35)$ & $<0.001$ & $0.25(0.10-0.64)$ & 0.004 \\
\hline
\end{tabular}

Notes: Model I, adjusted for age, gender and BMI; Model 2, adjusted for age, gender, BMI, systolic pressure, diastolic pressure, IADL, GDS, sleep duration, IPAQ, Illiterate, widow, living alone, job category, monthly income, intercourse of relatives, diabetes, hypertension, hyperlipidemia, Hepatic disease.

Abbreviation: TUGT, timed up and go test.

Table 3 Multivariate Linear Regression Analysis of the Association Between Grip Strength and Cognitive Domains

\begin{tabular}{|c|c|c|c|c|c|c|}
\hline \multirow[t]{2}{*}{ Variables } & \multicolumn{2}{|c|}{ Unadjusted } & \multicolumn{2}{|c|}{ Model I } & \multicolumn{2}{|c|}{ Model 2} \\
\hline & $\beta$ & $\mathbf{P}$ & $\beta$ & $P$ & $\boldsymbol{\beta}$ & $P$ \\
\hline MMSE score & 0.298 & $<0.001$ & 0.238 & $<0.001$ & 0.120 & 0.002 \\
\hline \multicolumn{7}{|l|}{ MMSE subscores } \\
\hline Orientation to time & 0.208 & $<0.001$ & 0.162 & $<0.001$ & 0.089 & 0.033 \\
\hline Orientation to place & 0.262 & $<0.001$ & 0.222 & $<0.001$ & 0.064 & 0.113 \\
\hline Registration & 0.131 & $<0.001$ & 0.096 & 0.003 & 0.097 & 0.022 \\
\hline Attention and calculation & 0.216 & $<0.001$ & 0.180 & $<0.001$ & 0.071 & 0.086 \\
\hline Recall & 0.103 & 0.001 & 0.071 & 0.029 & 0.077 & 0.073 \\
\hline Language & 0.282 & $<0.001$ & 0.164 & $<0.001$ & 0.121 & $<0.001$ \\
\hline
\end{tabular}

Notes: Model I, adjusted for age, gender and BMI; Model 2, adjusted for age, gender, BMI, systolic pressure, diastolic pressure, IADL, GDS, sleep duration, IPAQ, Illiterate, widow, living alone, job category, monthly income, intercourse of relatives, diabetes, hypertension, hyperlipidemia, Hepatic disease.

Abbreviation: MMSE, Mini-mental State Examination.

Table 4 Multivariate Linear Regression Analysis of the Association Between TUGT and Cognitive Domains

\begin{tabular}{|l|l|l|l|l|l|l|}
\hline Variables & \multicolumn{2}{l|}{ Unadjusted } & \multicolumn{2}{l|}{ Model I } & \multicolumn{2}{l|}{ Model 2} \\
\cline { 2 - 6 } & $\boldsymbol{\beta}$ & $\boldsymbol{P}$ & $\boldsymbol{\beta}$ & $\boldsymbol{P}$ & $\boldsymbol{\beta}$ \\
\hline MMSE score & -0.334 & $<0.001$ & -0.247 & $<0.001$ & -0.182 & \\
MMSE subscores & & & & & \\
Orientation to time & -0.209 & $<0.001$ & -0.141 & $<0.001$ & -0.103 \\
Orientation to place & -0.237 & $<0.001$ & -0.168 & $<0.001$ & -0.126 & 0.003 \\
Registration & -0.213 & $<0.001$ & -0.173 & $<0.001$ & -0.133 & $<0.001$ \\
Attention and calculation & -0.229 & $<0.001$ & -0.173 & $<0.001$ & -0.134 & $<0.001$ \\
Recall & -0.140 & $<0.001$ & -0.097 & 0.004 & -0.062 & $<0.001$ \\
Language & -0.335 & $<0.001$ & -0.255 & $<0.001$ & -0.156 \\
\hline
\end{tabular}

Notes: Model I, adjusted for age, gender and BMI; Model 2, adjusted for age, gender, BMI, systolic pressure, diastolic pressure, IADL, GDS, sleep duration, IPAQ, Illiterate, widow, living alone, job category, monthly income, intercourse of relatives, diabetes, hypertension, hyperlipidemia, Hepatic disease.

Abbreviation: MMSE, Mini-mental State Examination.

Further, the relationships between physical performance and specific cognitive function were analyzed. The grip strength was found to correlate with the overall cognition, time orientation, registration, and language. However, walking speed and TUGT correlated with all areas of cognition, except recall.

\section{Relationship Between Grip Strength and $\mathrm{MCl}$}

This study showed that grip strength correlated with MCI. Further analysis of the relationship between grip strength and overall cognition and various cognitive domains revealed that the grip strength correlated with overall cognition, time orientation, immediate recall, and language, but grip strength and orientation did not correlate with place, attention and calculation, and recall. Studies found $^{24}$ that every $5 \mathrm{~kg}$ higher handgrip strength was associated with lower odds ( $\mathrm{OR}=0.97,95 \% \mathrm{CI}=0.93-$ 0.99) for both future cognitive impairment and worse cognitive impairment. However, the relationship between grip strength and overall cognitive and its relationship with 
Table 5 Multivariate Linear Regression Analysis of the Association Between 4-Meter Walking Speed and Cognitive Domains

\begin{tabular}{|c|c|c|c|c|c|c|}
\hline \multirow[t]{2}{*}{ Variables } & \multicolumn{2}{|c|}{ Unadjusted } & \multicolumn{2}{|c|}{ Model I } & \multicolumn{2}{|c|}{ Model 2} \\
\hline & $\boldsymbol{\beta}$ & $P$ & $\boldsymbol{\beta}$ & $P$ & $\boldsymbol{\beta}$ & $P$ \\
\hline MMSE score & 0.343 & $<0.001$ & 0.219 & $<0.001$ & 0.153 & $<0.001$ \\
\hline \multicolumn{7}{|l|}{ MMSE subscores } \\
\hline Orientation to time & 0.217 & $<0.001$ & 0.121 & $<0.001$ & 0.082 & 0.021 \\
\hline Orientation to place & 0.236 & $<0.001$ & 0.125 & $<0.001$ & 0.078 & 0.023 \\
\hline Registration & 0.190 & $<0.001$ & 0.141 & $<0.001$ & 0.096 & 0.008 \\
\hline Attention and calculation & 0.234 & $<0.001$ & 0.149 & $<0.001$ & 0.109 & 0.002 \\
\hline Recall & 0.144 & $<0.001$ & 0.095 & 0.007 & 0.062 & 0.089 \\
\hline Language & 0.359 & $<0.001$ & 0.252 & $<0.001$ & 0.154 & $<0.001$ \\
\hline
\end{tabular}

Notes: Model I, adjusted for age, gender and BMI; Model 2, adjusted for age, gender, BMI, systolic pressure, diastolic pressure, IADL, GDS, sleep duration, IPAQ, Illiterate, widow, living alone, job category, monthly income, intercourse of relatives, diabetes, hypertension, hyperlipidemia, Hepatic disease.

Abbreviation: MMSE, Mini-mental State Examination.

other cognitive domains need to be further observed. Other studies showed that grip strength was not associated with MCI. ${ }^{9}$ A longitudinal study ${ }^{25}$ that followed the grip strength and cognition for more than 20 years found that the grip strength had an effect on the processing speed before the age of 65 years, and the grip strength was related to memory, processing speed, and spatial ability after 65 years, indicating that it was due to a change in lifestyle. Around age 65 years, the aging process intensifies for many and starts to accelerate, resulting in greater individual differences and also qualitative changes affecting the relationship between the variables. Some researchers argued that this might be due in part to a common biologic process underlying age-related declines in both physical and cognitive functioning. ${ }^{26}$ Physical and cognitive functioning are both indicators of biologic aging. The temporal relationship between cognitive and physical performance differs across domains and age, suggesting a specific rather than a general relationship. This emphasizes the importance of repeated measurements on different domains and encourages efforts on the development of domain and age-specific interventions.

Several possible explanations exist for the mechanism underlying the relationship between grip strength and cognitive function. One possible explanation is that cognitive impairment may only show physical inactivity in the earliest stage, leading to a loss of muscle mass and strength over time. At the same time, since participation in exercise and activity is related to an increase in brain plasticity and improvement in cognitive function, ${ }^{27}$ it suggests that lifestyle factors may be the intermediary factors to offset a potential decline in cognitive ability. Another explanation is that both cognitive and muscular systems rely on the nervous system to perform functions, so nervous system damage, for example, can lead to defects in both areas as a result of chronic inflammation. ${ }^{28}$ Further studies are required to clarify the neurophysiological basis of the relationship between muscle strength and cognitive function.

\section{Relationship Between TUGT and $\mathrm{MCl}$}

This study found a correlation between TUGT and MCI (OR $=1.08,95 \% \mathrm{CI}=1.03-1.13$ ). This was consistent with the results of previous studies. ${ }^{29}$ Further analysis revealed that TUGT correlated with overall cognition and various cognitive domains, except recall. One study found that generally older adults with MCI took a longer time to accomplish TUGT; however, the interaction (gender $\times$ age $\times$ cognitive status) was not statistically significant. ${ }^{11}$ Several studies found that slower TUGT was associated with cognitive language, poor attention and computing skills. ${ }^{30}$ This was consistent with the results of the present study. Greene ${ }^{10}$ found that TUGT could be used as a longitudinal monitoring tool to identify individuals at risk of cognitive decline. Donoghue et al found little evidence of a relationship between baseline mobility and decline in cognitive function in this highfunctioning community-dwelling sample. ${ }^{12}$ Previous studies $^{31}$ showed that the hippocampus was a key brain region for gait control in both physiological and pathological (early cognitive decline) aging, and longer TUGT was associated with lower hippocampal volume; the strength of the relationship between hippocampus and TUGT was the highest for the hippocampus compared with other brain regions. Examining the early modifications of MCI status related to 
the brain structures involved in gait control improved the understanding of the neuropathological mechanisms underlying gait disorders in aging. Although the TUGT test was simple, it required the cooperation of many systems, and the process was complex, especially in older adults with cognitive impairment. Therefore, more longitudinal studies are needed to explore the relationship between TUGT and MCI in the future.

\section{Relationship Between 4-m Walking Speed and $\mathrm{MCl}$}

A correlation was found between 4-m walking speed and MCI $(\mathrm{OR}=0.25,95 \% \mathrm{CI}=0.10-0.64)$ in the present study. Many studies showed that slow gait speed was associated with an increased risk of incident $\mathrm{MCI}^{9}$ and $\mathrm{AD} .^{32} \mathrm{~A}$ well-characterized cohort study found that a longitudinal measure of gait speed decline over 14 years predicted later cognitive impairment (adjudicated MCI or dementia) in an initially healthy sample of older adults. ${ }^{33}$ Another study $^{34}$ showed that individuals with MCI had significantly slower walking speed and worse geriatric depression scale compared with non-MCI, which was similar to the results of this study.

Further analysis revealed that 4-m walking speed was related to overall cognition and various cognitive domains, except recall. McGough et al found that slow gait was associated with registration, attention, calculation, and executive performance. $^{35}$ Another study found a relationship between slower gait speed and worse baseline performance on measures of memory, attention, information processing speed, and verbal fluency. ${ }^{9}$ These findings indicated that future studies should focus on the changes in walking speed in older adults, and more welldesigned cohort studies need to be carried out to verify the relationship between walking speed and MCI. A study ${ }^{36}$ showed that a smaller volume of the prefrontal area might contribute to slower gait through slower information processing. Evidence from previous studies ${ }^{37}$ indicated that the volume of the prefrontal area and information processing speed were modifiable late in life. Therefore, longitudinal studies are warranted to examine the casual relationship between focal brain atrophy with slowing in information processing and gait. Further studies should investigate mechanisms and early intervention strategies that assist older adults with MCI to maintain function and independence.

\section{Strengths and Limitations}

This study had a number of strengths. First, this novel study examined the relationship between physical performance and MCI in Chinese older adults. Second, this study was also the first to examine the relationship between physical performance and specific cognitive functions. Recruited participants from a suburban area led a more physically active lifestyle, differentiating them from participants in other geographical areas. However, the study also had certain limitations. First, all participants in the present study were relatively healthy. Individuals unable to participate in the free annual national physical examination were excluded. This choice could constitute a selective survival and a healthy selection bias. Second, this was a cross-sectional study, and therefore it was difficult to find out the causal relationship between them. Sample sizes and years of follow-up need to be increased in future studies to verify the relationship. Third, although the MMSE could be used for the initial classification of patients with $\mathrm{MCI}$, this brief cognitive test had some shortcomings in defining MCI. Adding some specific indicators, such as functional magnetic resonance imaging, should be considered in the future.

\section{Conclusions}

The physical performance (grip strength, TUGT, and 4-m walking speed) was found to correlate with MCI. Further analysis showed that the grip strength was associated with overall cognition, time orientation, recall, and language, while TUGT and 4-m walking speed were associated with overall cognition and various cognitive domains, except recall. The older adults with lower grip strength, lower walking speed, and slower TUGT might be the focus of MCI, and cognitive screening should be conducted in time. The grip strength, walking speed, and TUGT might be significant indicators for the early identification of MCI. Establishing the screening and evaluation of the physical performance of older adults aged more than 65 years in the Chinese community is necessary for the early detection and intervention of MCI.

\section{Acknowledgments}

The authors thank Peijuan Jin and Xiaoyue $\mathrm{Gu}$ from the Chongming public health center for providing place and organization. Yuewen Liu and Weibo Ma are co-first authors for this study. 


\section{Funding}

This work was supported by Shanghai Sailing Program (20YF1418200), the Hygiene and Health Commission of Shanghai municipality (2019HP76) and the funding of Youth Fund Project of Research Planning Foundation on Humanities and Social Sciences of the Ministry of Education (20YJCZH001).

\section{Disclosure}

The authors declare that they have no conflicts of interest for this work.

\section{References}

1. Winblad B, Palmer K, Kivipelto M, et al. Mild cognitive impairment-beyond controversies, towards a consensus: report of the International Working Group on Mild Cognitive Impairment. $J$ Intern Med. 2004;256(3):240-246. doi:10.1111/j.13652796.2004.01380.x

2. Lopez ME, Turrero A, Cuesta P, et al. Searching for primary predictors of conversion from mild cognitive impairment to alzheimer's disease: a multivariate Follow-Up study. J Alzheimers Dis. 2016;52 (1):133-143. doi:10.3233/JAD-151034

3. Eshkoor SA, Hamid TA, Mun CY, et al. Mild cognitive impairment and its management in older people. Clin Interv Aging. 2015;10:687-693. doi:10.2147/CIA.S73922

4. Hebert LE, Scherr PA, Bienias JL, et al. Alzheimer disease in the US population: prevalence estimates using the 2000 census. Arch Neurol. 2003;60(8):1119-1122. doi:10.1001/archneur.60.8.1119

5. Montero-Odasso M, Oteng-Amoako A, Speechley M, et al. The motor signature of mild cognitive impairment: results from the gait and brain study. J Gerontol a Biol Sci Med Sci. 2014;69 (11):1415-1421. doi:10.1093/gerona/glu155

6. Allali G, van der Meulen M, Beauchet $\mathrm{O}$, et al. The neural basis of age-related changes in motor imagery of gait: an fMRI study. $J$ Gerontol a Biol Sci Med Sci. 2014;69(11):1389-1398. doi:10.1093/gerona/glt207

7. Blumen HM, Holtzer R, Brown LL, et al. Behavioral and neural correlates of imagined walking and walking-while-talking in the elderly. Hum Brain Mapp. 2014;35(8):4090-4104. doi:10.1002/ hbm. 22461

8. Holtzer R, Epstein N, Mahoney JR, et al. Neuroimaging of mobility in aging: a targeted review. J Gerontol a Biol Sci Med Sci. 2014;69 (11):1375-1388. doi:10.1093/gerona/glu052

9. Hooghiemstra AM, Ramakers IHGB, Sistermans N, et al. Gait speed and grip strength reflect cognitive impairment and are modestly related to incident cognitive decline in memory clinic patients with subjective cognitive decline and mild cognitive impairment: findings from the 4C study. J Gerontol Ser A. 2017;72(6):846-854. doi:10.1093/gerona/glx003

10. Greene BR, Kenny RA. Assessment of cognitive decline through quantitative analysis of the timed up and go test. Ieee T Bio-Med Eng. 2012;59(4):988-995. doi:10.1109/TBME.2011.2181844

11. Ibrahim A, Singh D, Shahar S. 'Timed Up and Go' test: age, gender and cognitive impairment stratified normative values of older adults. PLoS One. 2017;12(10):e185641. doi:10.1371/journal.pone.0185641

12. Donoghue O, Feeney J, O'Leary N, et al. Baseline mobility is not associated with decline in cognitive function in healthy Community-Dwelling older adults: findings from the irish longitudinal study on ageing (TILDA). Am J Geriatric Psychiatry. 2018;26 (4):438-448. doi:10.1016/j.jagp.2017.11.006
13. Kobayashi-Cuya KE, Sakurai R, Suzuki H, et al. Observational evidence of the association between handgrip strength, hand dexterity, and cognitive performance in Community-Dwelling older adults: a systematic review. J Epidemiol. 2018;28(9):373-381. doi:10.2188/ jea.JE20170041

14. Arevalo-Rodriguez I, Smailagic N, Roque IFM, et al. Mini-Mental State Examination (MMSE) for the detection of Alzheimer's disease and other dementias in people with mild cognitive impairment (MCI). Cochrane Database Syst Rev. 2015;(3):D10783.

15. Folstein MF, Folstein SE, McHugh PR. Mini-mental state". A practical method for grading the cognitive state of patients for the clinician. J Psychiatr Res. 1975;12(3):189-198.

16. Zhang J, Tang G, Xie H, et al. Higher adiposity is associated with slower cognitive decline in hypertensive patients: secondary analysis of the china stroke primary prevention trial. $J$ Am Heart Assoc. 2017;6:10. doi:10.1161/JAHA.117.005561

17. Lawton MP, Brody EM. Assessment of older people: self-maintaining and instrumental activities of daily living. Gerontologist. 1969;9 (3):179-186. doi:10.1093/geront/9.3_Part_1.179

18. Petersen RC, Smith GE, Waring SC, et al. Mild cognitive impairment: clinical characterization and outcome. Arch Neurol. 1999;56 (3):303-308. doi:10.1001/archneur.56.3.303

19. Zhang MY, Katzman R, Salmon D, et al. The prevalence of dementia and Alzheimer's disease in Shanghai, China: impact of age, gender, and education. Ann Neurol. 1990;27(4):428-437. doi:10.1002/ ana.410270412

20. Nagamatsu LS, Chan A, Davis JC, et al. Physical activity improves verbal and spatial memory in older adults with probable mild cognitive impairment: a 6-month randomized controlled trial. J Aging Res. 2013;2013:861893. doi:10.1155/2013/861893

21. Shimada H, Suzuki T, Suzukawa M, et al. Performance-based assessments and demand for personal care in older Japanese people: a cross-sectional study. BMJ Open. 2013;3:4. doi:10.1136/bmjopen2012-002424

22. Jiang CQ, Xu L, Lam TH, et al. [Effect of physical activity strength on the diabetes mellitus prevalence in the elderly under the influence of International Physical Activity Questionnaire]. Zhonghua Liu Xing Bing Xue Za Zhi. 2009;30(5):462-465. Chinese.

23. Xue J, Li J, Liang J, et al. The prevalence of mild cognitive impairment in china: a systematic review. Aging Dis. 2018;9(4):706. doi:10.14336/AD.2017.0928

24. McGrath R, Vincent BM, Hackney KJ, et al. The longitudinal associations of handgrip strength and cognitive function in aging americans. $J$ Am Med Dir Assoc. 2019;S1525-8610 (19):30648-30649.

25. Sternang O, Reynolds CA, Finkel D, et al. Grip strength and cognitive abilities: associations in old age. J Gerontol B Psychol Sci Soc Sci. 2016;71(5):841-848. doi:10.1093/geronb/gbv017

26. Clouston SAP, Brewster P, Kuh D, et al. The dynamic relationship between physical function and cognition in longitudinal aging cohorts. Epidemiol Rev. 2013;35(1):33-50. doi:10.1093/epirev/ mxs004

27. Erickson KI, Weinstein AM, Lopez OL. Physical activity, brain plasticity, and Alzheimer's disease. Arch Med Res. 2012;43 (8):615-621. doi:10.1016/j.arcmed.2012.09.008

28. Fritz NE, McCarthy CJ, Adamo DE. Handgrip strength as a means of monitoring progression of cognitive decline - a scoping review. Ageing Res Rev. 2017;35:112-123. doi:10.1016/j.arr.2017.01.004

29. Lee S, Han J, Jin Y, et al. Poor physical fitness is independently associated with mild cognitive impairment in elderly Koreans. Biol Sport. 2016;33(1):57-62. doi:10.5604/20831862.1185889

30. Donoghue OA, Horgan NF, Savva GM, et al. Association between timed up-and-go and memory, executive function, and processing speed. J Am Geriatr Soc. 2012;60(9):1681-1686. doi:10.1111/ j.1532-5415.2012.04120.x 
31. Allali G, Annweiler C, Predovan D, et al. Brain volume changes in gait control in patients with mild cognitive impairment compared to cognitively healthy individuals; GAIT study results. Exp Gerontol. 2016;76:72-79. doi:10.1016/j.exger.2015.12.007

32. Gillain S, Dramé M, Lekeu F, et al. Gait speed or gait variability, which one to use as a marker of risk to develop Alzheimer disease? A pilot study. Aging Clin Exp Res. 2016;28(2):249-255. doi:10.1007/ s40520-015-0392-6

33. Rosso AL, Verghese J, Metti AL, et al. Slowing gait and risk for cognitive impairment: the hippocampus as a shared neural substrate. Neurology. 2017;89(4):336-342. doi:10.1212/WNL.0000000000004153

34. Kume Y, Takahashi T, Itakura Y, et al. Characteristics of mild cognitive impairment in northern japanese Community-Dwellers from the ORANGE registry. J Clin Med. 2019;8(11):1937. doi:10.3390/ jcm8111937
35. McGough EL, Cochrane BB, Pike KC, et al. Dimensions of physical frailty and cognitive function in older adults with amnestic mild cognitive impairment. Ann Phys Rehabil Med. 2013;56(5):329-341. doi:10.1016/j.rehab.2013.02.005

36. Rosano C, Studenski SA, Aizenstein HJ, et al. Slower gait, slower information processing and smaller prefrontal area in older adults. Age Ageing. 2012;41(1):58-64. doi:10.1093/ageing/afr113

37. Rosano C, Venkatraman VK, Guralnik J, et al. Psychomotor speed and functional brain MRI 2 years after completing a physical activity treatment. J Gerontol a Biol Sci Med Sci. 2010;65A(6):639-647. doi:10.1093/gerona/glq038

\section{Publish your work in this journal}

Clinical Interventions in Aging is an international, peer-reviewed journal focusing on evidence-based reports on the value or lack thereof of treatments intended to prevent or delay the onset of maladaptive correlates of aging in human beings. This journal is indexed on PubMed Central, MedLine, CAS, Scopus and the Elsevier
Bibliographic databases. The manuscript management system is completely online and includes a very quick and fair peer-review system, which is all easy to use. Visit http://www.dovepress.com/ testimonials.php to read real quotes from published authors. 